\title{
First light and results on EBL2
}

Norbert Koster, Edwin te Sligte, Alex Deutz, Freek Molkenboer, Pim Muilwijk, et al. 


\title{
First light and results on EBL2
}

\author{
Norbert Koster*, Edwin te Sligte, Alex Deutz, Freek Molkenboer, Pim Muilwijk, Peter van der \\ Walle, Wouter Mulckhuyse, Bjorn Nijland, Peter kerkhof, Michel van Putten \\ TNO, Stieltjesweg 1, 2628 CK Delft, The Netherlands
}

\begin{abstract}
Recently TNO has established EBL2; an exposure and analysis facility for testing EUV optics, reticles and pellicles under relevant EUV scanner and source conditions. The facility and EUV source complies with the ASML power roadmap of EUV systems up to a power of $500 \mathrm{~W}$ IF. This enables life time testing of EUV optics, reticles and pellicles under conditions which are not yet available to industry, helping the industry in preparing for HVM production.

The EBL2 facility consists of a EUV source, collector optics, exposure chamber, XPS chamber, and automated sample handling. The exposure chamber has capabilities for plasma analysis, imaging ellipsometry for in-situ analysis of the sample under radiation, photodiodes for power measurements and a scintillator disk for spot profiles. It is possible to insert spectral purity filters and apertures in the beam line for wavelength tuning and beam shaping. The source is Sn fueled DPP source made by our partner Ushio and is based on the proven technology from the ASML AD-tools, providing a similar spectrum and pulse shape as used in the ASML NXE scanners. We show the results of first light obtained in December 2016. The XPS is capable of handling and analyzing full reticles and data on the obtained surface sensitivity and imaging quality will be shown.
\end{abstract}

Keywords: EUV exposure, mask, pellicle, XPS analysis, metrology, handling, contamination control

\section{INTRODUCTION}

EUV is approaching its insertion point for high volume manufacturing as EUV sources and EUV lithography tools become increasingly mature products ${ }^{[1]}$. While the NXE source power roadmap has grown up to powers of $500 \mathrm{~W}$ and some even dream beyond this, there is a need to investigate the effects of these kind of powers on optics, reticles and pellicles. With the maturing of the EUV technology there is also an increased need to develop a reticle and pellicle infra structure. Many issues remain to be addressed in these fields, and testing will play an indispensable part in resolving these issues.

TNO International Centre for Contamination Control (ICCC) is dedicated to developing the highest standards and practices in contamination control, for preventing and eliminating both particle contamination and molecular contamination in space and semiconductor applications. In 2005, TNO and Carl Zeiss SMT GmbH jointly established the unique EBL test facility to further the development of EUV technology. EBL contains an EUV Beam Line, in which samples can be exposed to EUV irradiation in a controlled environment. Attached to the Beam Line is an XPS system, which can be reached via an in-vacuum sample transfer system. This enables surface analysis of exposed samples without breaking vacuum. The compound instrument is used to develop and validate optics lifetime strategies for ASML EUV scanners ${ }^{[2]}$.

As our old EBL is no longer capable of keeping up with the power roadmap and is also not capable of handling larger samples like reticles and a new system was designed, called EBL2. The concept study was described in Ref. ${ }^{[3]}$. The system was realized in close collaboration with our technology partners Ushio Inc for the Sn fueled DPP EUV source and ASYS Automatic Systems GmbH \& Co. KG for both the vacuum and atmospheric handler. EBL2 will be able to expose a diversity of samples up to full reticles (including pellicles) at several locations on the sample. The power on the

*Corresponding author: norbert.koster@tno.nl, TNO the Netherlands, +31 888666339

Photomask Japan 2017: XXIV Symposium on Photomask and Next-Generation Lithography

Mask Technology, edited by Kiwamu Takehisa, Proc. of SPIE Vol. 10454, 1045400

(C) 2017 SPIE $\cdot$ CCC code: $0277-786$ X/17/\$18 $\cdot$ doi: $10.1117 / 12.2279025$

Proc. of SPIE Vol. 10454 1045400-1 
sample is compliant with the roadmap up to at least $250 \mathrm{~W}$ IF for scanners and the gas environment in the exposure chamber can mimic scanner and source conditions. A summary of the performance of EBL2 is listed in Table 1.

Table 1: comprehensive overview of EBL2 performance

\begin{tabular}{|l|l|}
\hline Power & $>1 \mathrm{~W}$ in $2 \% \mathrm{BW} @ 13.5 \mathrm{~nm} @ 3 \mathrm{kHz}$ (“IB”) ( 10 W 10-20 nm OoB) \\
\hline Power density & $>1 \mathrm{~W} / \mathrm{mm}^{2} \mathrm{IB}$ in focus \\
\hline Spot size & $1-30 \mathrm{~mm}$ diameter (power density scales) \\
\hline Rep rate & $1 \mathrm{~Hz}-10 \mathrm{kHz}$ (standard $3 \mathrm{kHz})$ \\
\hline Sample size & Max $152 \times 152 \times 20 \mathrm{~mm}$ (EUV mask + pellicle possible) \\
\hline Dose control & $<20 \%$ in free running experiment \\
\hline Uninterrupted exposure time & $>100$ hours \\
\hline
\end{tabular}

\section{SYSTEM LAYOUT}

A full system description can be found in ref [4] and [5], in this part a short summary of EBL2 system will be given. EBL2 consists of a) Ushio EUV source mounted on a track for easy maintenance access, b) collector module consisting of two elliptical grazing incidence mirrors with intermediate focus, c) exposure chamber with in-situ diagnostics, d) sample handler with vacuum and atmospheric handler, dual pod opener, storage capability and cleaning chamber, and e) XPS system capable of handling reticles for surface analysis of EBL2 and other samples.

The Ushio EUV source ${ }^{[6]}$ has a mechanical debris shield to mitigate any Sn debris originating from the source towards the collector optics. This shield is based on the proven technology as used in the ASML AD tools, which have been in use for a number of years at CNSE (Albany, USA) and Imec (Leuven, Be). The source has an increased NA to be able to collect more light in the collector optics. The collector optics are Ru coated grazing incidence elliptical mirrors. The main collector (M1) collects the light from the source and focusses this in the intermediate focus (IF), located approximately at 2/3 distance away from the source. The second mirror (M2) then focusses the light on the sample in the exposure chamber. The IF interface is also used as a pumping aperture to suppress any gasses flowing from the source to the exposure chamber and vice versa. In the collector module it is possible to insert apertures and filters to modify the beam with respect to spectrum and profile. Defocusing of the light on the sample to create a larger spot is established by moving collector module and source on the track to the left. The connection between collector and exposure chamber is a long flexible bellow to accommodate moving while under vacuum.

The exposure chamber has in-situ diagnostics with an imaging ellipsometer. This ellipsometer is also used to find markers on the samples and use those for navigation on the sample to the desired position. Moving the sample is accomplished by a hexapod stage system mounted to the bottom part of the EC. This hexapod drive system is mounted on the atmospheric side of the system and vacuum connection is established by a very large bellow. By doing so all the motors, encoders and manipulators are not in vacuum and easily accessible for maintenance. This hexapod drive system is delivered by Symetrie (Fr).

The chamber itself is fully metal sealed, electropolished and cleaned to a high degree of cleanliness. Actuation inside the chamber for gripping and flipping of the sample is done by pneumatically actuated bellows. This ensures cleanliness of the system as no motors or lubricants are used in vacuum. The chamber has a differentially pumped RGA to check cleanliness of the background and control the gasses admitted for partial pressures and composition. The stage is equipped with calibrated photodiodes to measure intensity and power of the EUV beam. In combination with a scintillator disk and a CCD camera spot size and intensity profiles can be measured. Both photodiodes and scintillator can be used to find the center of the EUV spot and relate this to stage coordinates. 


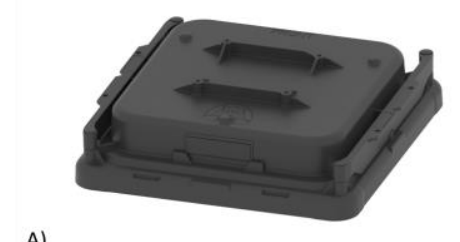

A)

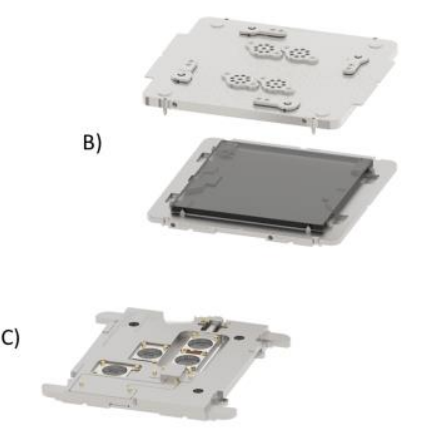

D)

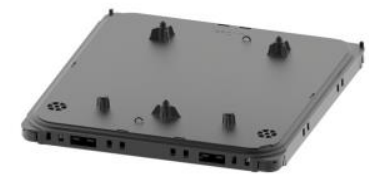

C)

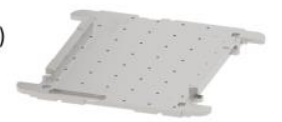

Figure 1: Types of sample holders used in EBL2. a) dual pod interface, b) reticle with inner pod, c) MLM holder with angle resolved capability, d) generic sample holder. All sample holders fit in dual pod outer pod

Both handlers and other modules are ASML NXE compatible with respect to reticle backside cleanliness. This enables exposures of reticles on EBL2 and afterwards wafer printing of the reticles on a ASML NXE scanner to verify the effect of high doses of EUV radiation on reticle performance. All samples are loaded using the dual pod EUV reticle interface. For samples other than reticles customized sample holders are available, see Figure 1 for possible configurations. Other type of holders are possible as long as they remain within the volume claim and have the same mechanical interface for gripping.

\section{INTEGRATION}

In early 2016 the construction of the dedicated cleanroom for EBL2 began. This cleanroom is placed in the Van Leeuwenhoek Laboratory (VLL) in Delft and is part of the facilities of TNO for ICCC for contamination control research for space and semiconductor applications. This laboratory is shared with the Technical University Delft (TUD) for semiconductor device manufacturing and quantum computing research. The cleanroom is an ISO class 8 cleanroom with a raised floor for cable and piping conduits below this floor. At the location of the load port a ISO class 4 area is created and in the atmospheric handler an ISO class 1area is established. A flow bench will be installed for manual handling of samples in a dedicated clean area. A separate grey room for technical installations like air conditioning and another grey room for roughing pumps, chillers and electronic cabinets is available. The clean room is in a separate area of the VLL with no access by outside parties to ensure client confidentiality on the dedicated samples EBL2 is going to handle.

Starting from April 2016 parts and modules started arriving and assembly of EBL2 modules began in separate laboratory areas. From June 2016 the cleanroom was accessible for TNO employees and integration of EBL2 began. First parts to enter the cleanroom are the exposure chamber and beamline frame elements. Next the vacuum handler arrived and was connected to the exposure chamber. In the meantime Factory Acceptance Testing (FAT) of the XPS and EUV source was under way and in October and November these modules arrived at TNO and were moved to the clean room for integration.

In the early stage of EBL2 we will work with only a small single segment M1 mirror providing limited power at sample level. In the second quarter of 2017 the single element M1 mirror will be replaced with a full size M1 collector and full power and intensity should then be available for users of EBL2. At the end of 2016 most essential parts of EBL2 were installed and alignment of collector optics and source could commence. In Figure 2 a full overview of the system is visible. 


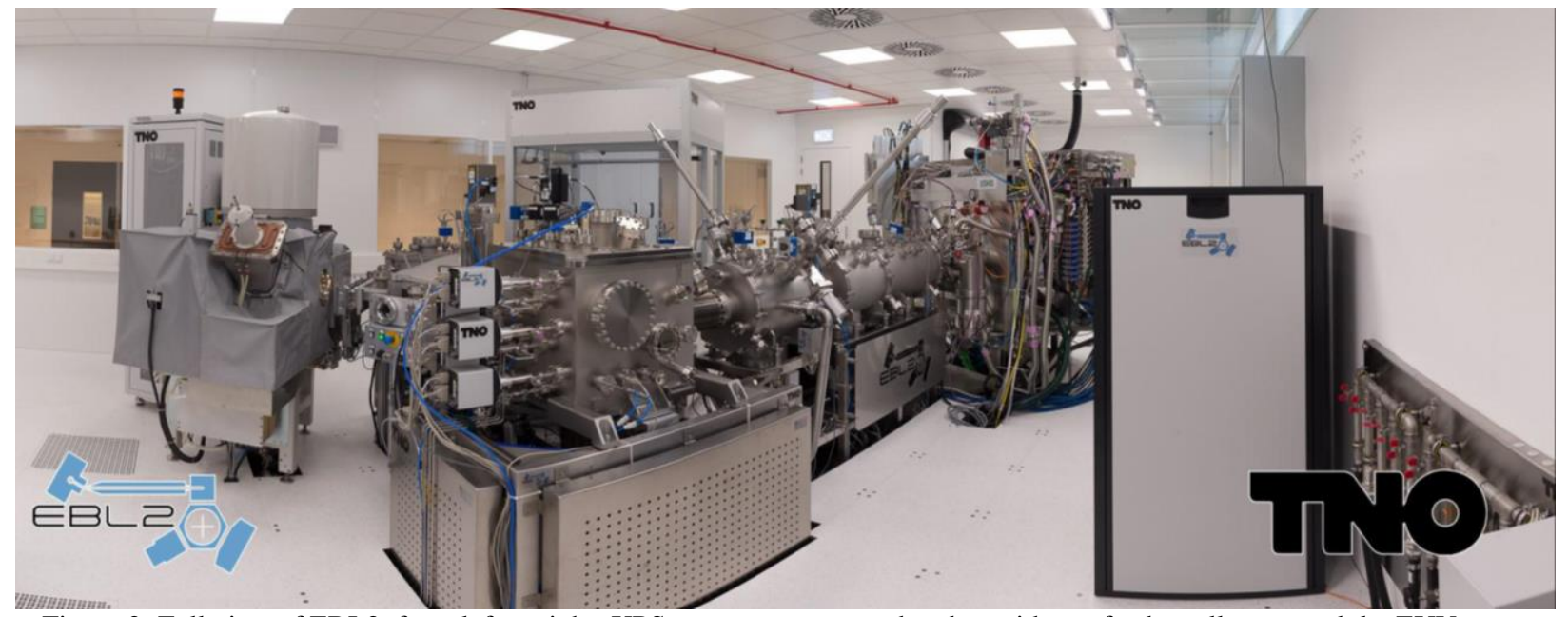

Figure 2: Full view of EBL2, from left to right: XPS system, exposure chamber with gas feeds, collector module, EUV source and in the back the vacuum and atmospheric handler.

\section{FIRST LIGHT}

In December 2016 the EBL2 beam line system consisting of source, collector and exposure chamber was connected. First rough alignment was done using visible light and lasers. When alignment was finished the system was evacuated and the source was heated for the first test runs.

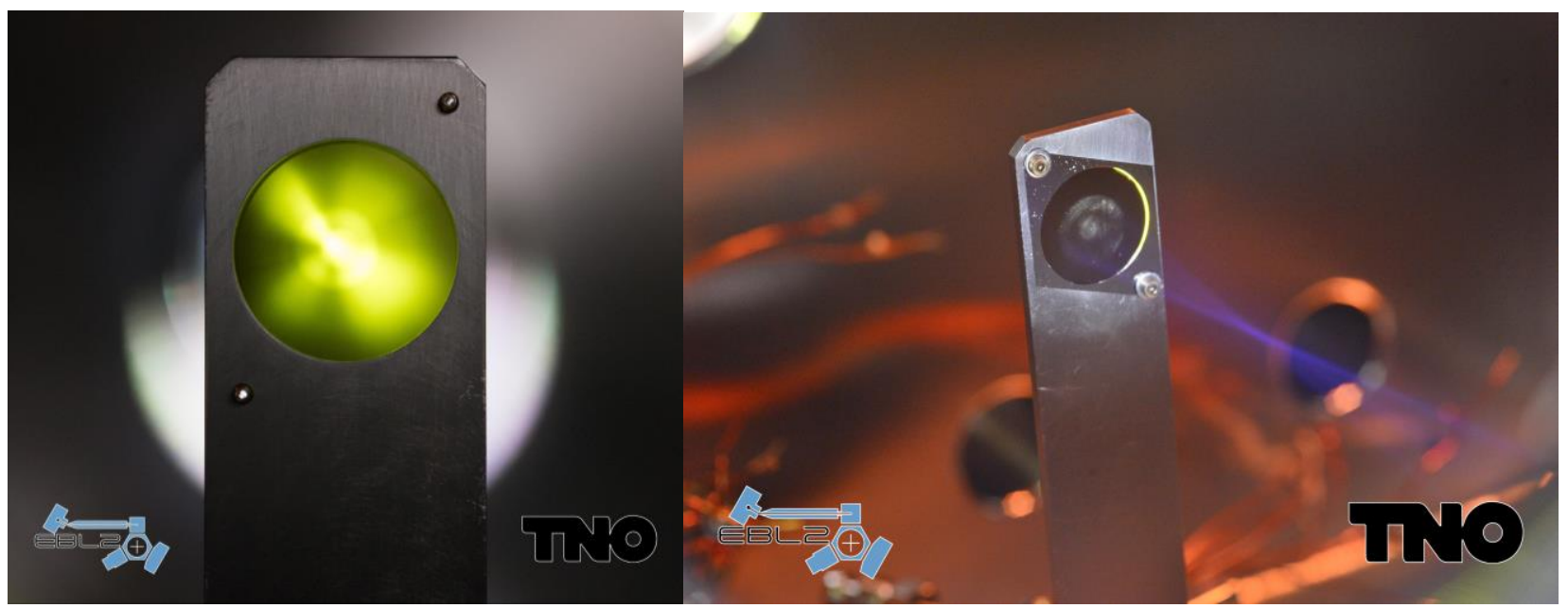

Figure 3: Left picture: First light on scintillator disk on sample position in the exposure chamber. Right picture: EUV induced plasma on the front side of the scintillator disk.

The team managed to get light through the complete optical column within one day after evacuating. In Figure 3 the results of the first light are shown. From the scintillator image on the left side it can be seen that there is side lobe on the spot. This is caused by the pumping aperture in the IF cone which is too small for the optical beam. Because the aperture is not a simple hole, but a cone tightly fitting around the optical beam it is possible to have EUV reflections as the EUV beam hits the surface at grazing incidence. The scintillator was placed behind the focus of the spot to prevent overheating. This can be seen in the right picture of Figure 3 where a focal spot can be observed in the plasma glow caused by the EUV radiation in a high pressure gas environment. This spot is approximately $6 \mathrm{~cm}$ in front of the scintillator disk. In the same picture the doughnut shape of the beam when out of focus can be seen on the scintillator disk. The plasma was created using Argon as a process gas with a pressure of about $1 \mathrm{~Pa}$. 


\section{XPS PERFORMANCE}

The XPS system is a modified Kratos Axis Nova tool. This system has been modified to be able to accept reticles and other samples like is shown in Figure 1. The maximum sample size is 152x152x20 mm, the height limit is caused by the distance between electron energy analyzer and the magnetic lens below the sample. The stage has a limited scan field, which is not capable of scanning a full reticle. To accommodate this it is possible to rotate the reticle in the atmospheric handler. The size of the scan field is shown in Figure 4. As can be seen from this figure about $62.5 \%$ of a reticle can be covered without the need of rotating the reticle and thus exposing the reticle to atmosphere. In case of surface sensitive experiments in the exposure chamber it is therefore necessary to limit the field of EUV exposure to the same field as can be scanned in the XPS.
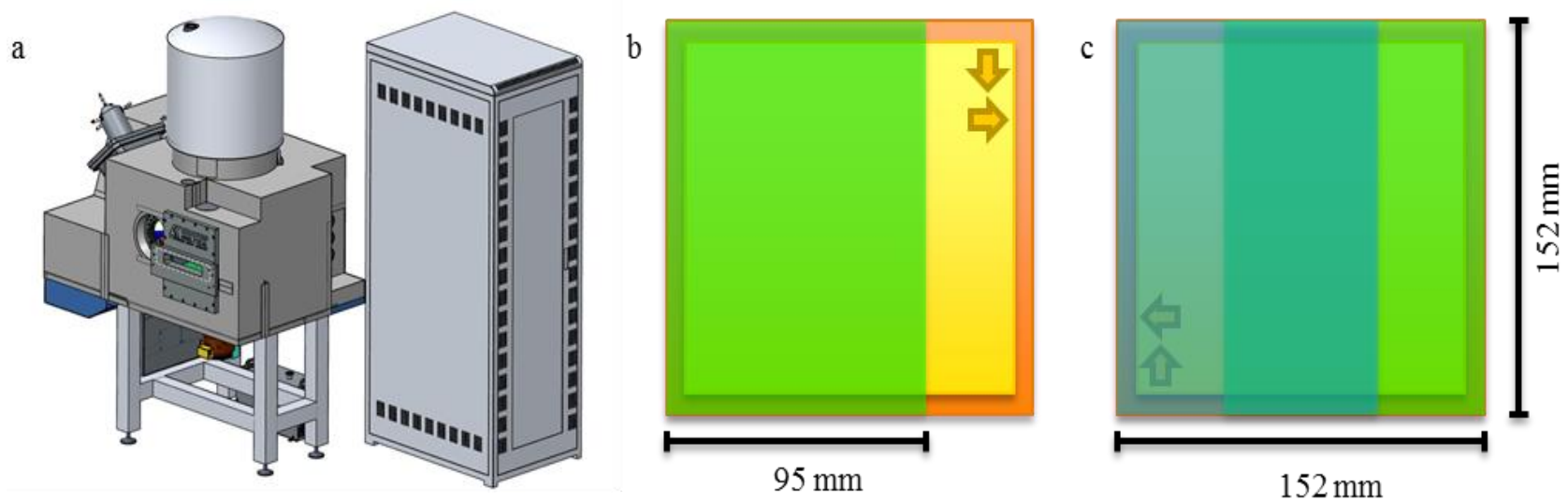

Figure 4: a) XPS subsystem on EBL2 b) Area coverage by XPS stage in one run and c) area coverage of reticle in two runs with rotating reticle in atmospheric handler

Besides the standard samples used for qualifying an XPS system, like Au grid, two special samples were prepared. The first sample is a EUV Multi Layer Mirror (MLM) with a Ru top coating, the second sample is a DUV reticle with $\mathrm{Cr}$ absorber with the EBL2 emblem etched into it at several sizes. The MLM is used to characterize angle resolved and depth profiling performance of the XPS. The reticle with emblem is used for testing the image mapping capability of the XPS. Using the visible light microscope in the XPS it is possible to locate the emblems and program the desired type of analysis to be performed by the XPS on that precise location. As both image mapping with XPS and depth profiling takes very long acquisition times these measurements are typically run over night in automatic mode.

For the MLM sample first the angle resolved mode was used and afterwards a depth profile using an Ar sputter gun was recorded. As the top layer of Ru is only about $1.5 \mathrm{~nm}$ thick and the Molybdenum Silicon multi layer stack has a period of about $6.7 \mathrm{~nm}$ it is essential to limit the ion energy to the lowest possible value, in our case $1 \mathrm{keV}$ as otherwise the multilayer gets intermixed an no depth signal is acquired. As can be seen from Figure 5 in the left figure the surface sensitivity increases with more gracing angles of the XPS. At gracing angle the intensity signal for Oxygen increases indicating a oxidized top surface of $\mathrm{Ru}$ due to exposure to ambient. In the right graph the Ru and $\mathrm{O}$ signal drops very fast after about 100 s, while the Si and Mo signals start to appear. After about 2000 s the Mo signal drops and the Si signal increases. This indicates that the Mo layer has been completely sputtered away and now the Si layer is being exposed.

As a second test the reticle with the etched emblem was loaded. First a visual image was captured using the visible light microscope in the measurement chamber and stitched together. XPS data was captured using a similar stitching method to cover the full emblem. Both visible light and XPS analysis can be seen in Figure 6. The length of the emblem is approximately $2.5 \mathrm{~mm}$ long. The XPS analysis shows the concentration of $\mathrm{Cr}$ and Si. The full XPS image was a 6 by 6 stitch of the field of the XPS in imaging mode. In both the visible and elemental image a fiber can be seen on the left side of the image. The fiber seems to have moved slightly during stage movements when changing from visible light microscopy to imaging mode. The resolution in imaging mode of the XPS is of the order of $0.1 \mathrm{~mm}$ as can be deducted from the missing fine details. 

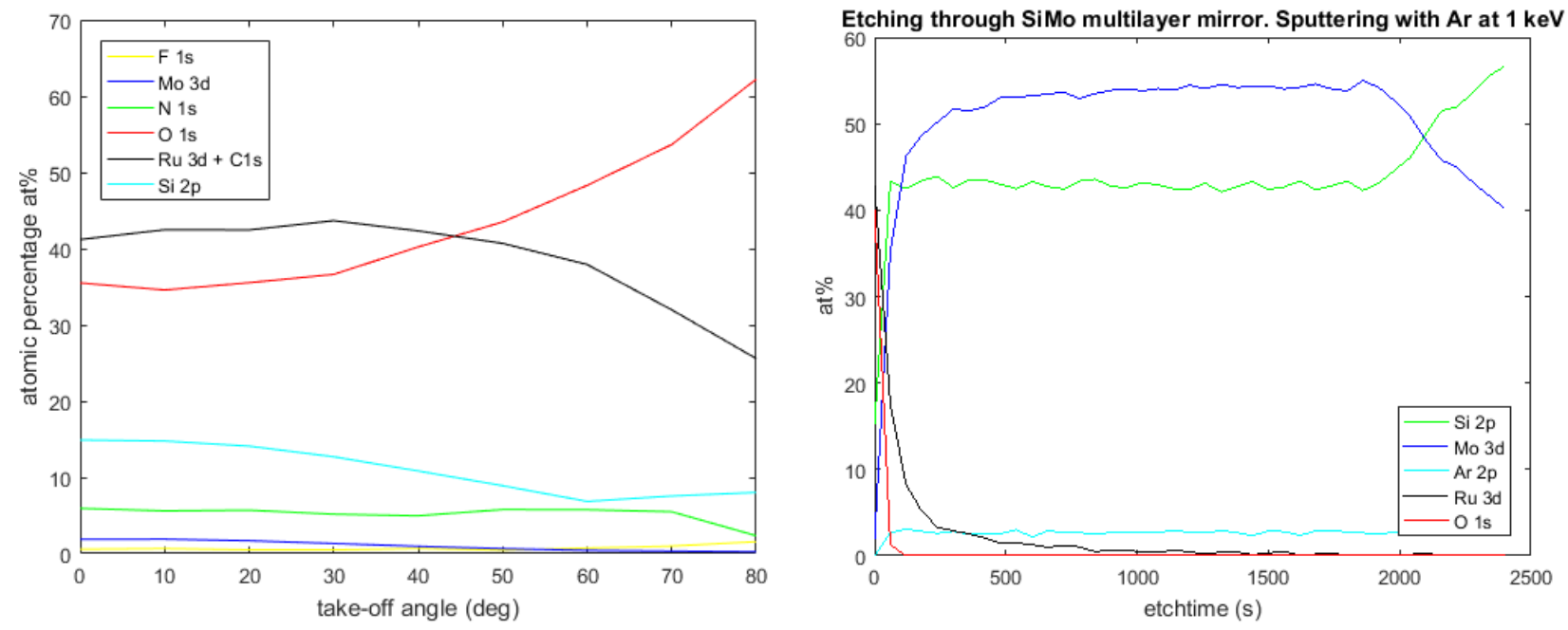

Figure 5: left) angle resolved measurement of MLM sample, right) depth profile of Ru cap layer and first Mo layer

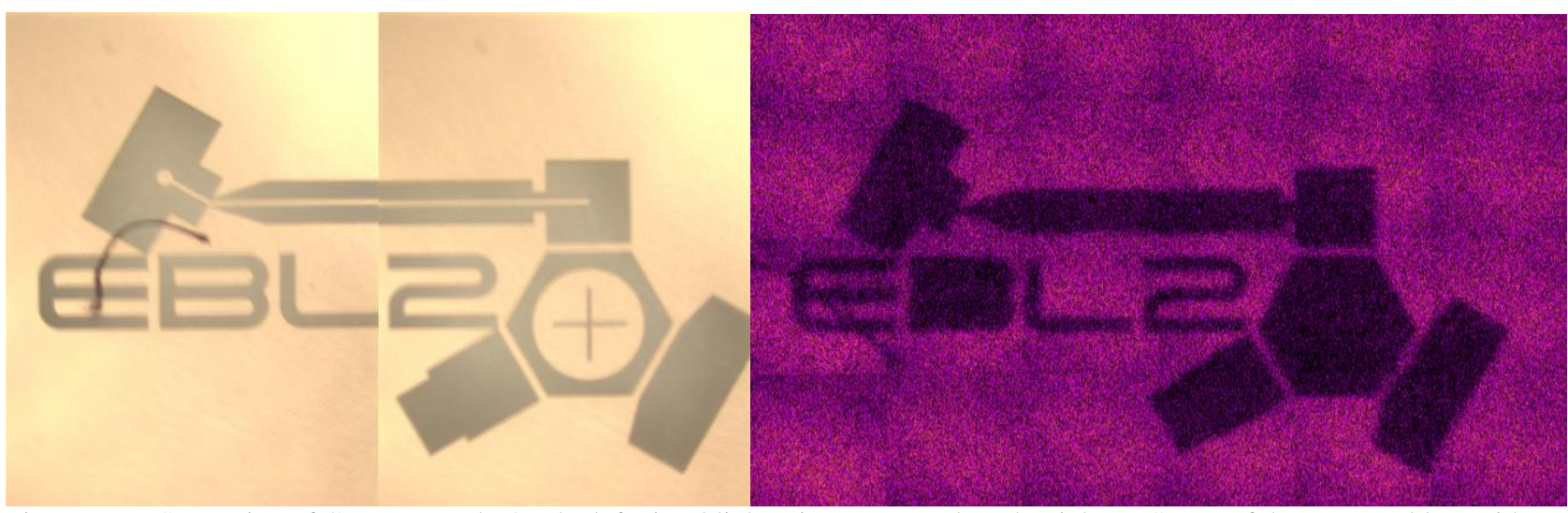

Figure 6: XPS mapping of Cr DUV mask. On the left visual light microscopy and on the right XPS map of the same emblem with a 6x6 stitch. The emblem size is approximately $2.5 \mathrm{~mm}$ long

\section{CONCLUSION}

The construction of EBL2 is going according to schedule and first light was achieved in December 2016. Ongoing activities on the system include installation and calibration of diagnostics like ellipsometer, photodiodes and RGA system. The XPS system is fully operational and can accept samples up to reticles from customers via automatic loading with the vacuum and atmospheric handlers using the dual pod interface. A major upgrade of the collector mirrors is expected to take place in May 2017 and then full EUV power and density is available on sample level. After the upgrade a full qualification program and first test runs on reticles will take place. The complete facility will be open for customers mid of 2017. Currently we are investigating the possibility to extend EBL2 with a EUV reflectometer to be able to perform reflection and transmission measurements on EUV optics, reticle and pellicles.

\section{ACKNOWLEDGEMENTS}

TNO would like thank its technology partners Ushio, Inc. and ASYS Automatic Systems GmbH \& Co. KG for the open and constructive collaboration in designing EBL2. TNO would also like to thank the potential users of EBL2 in helping with defining the right requirements and needs for EBL2. EBL2 is a TNO initiative and realization was made possible with funding from the European Union (SENATE and E450LMDAP project), Dutch ministry of Economic Affairs (NanoLabNL and Toekomstfonds). 


\section{REFERENCES}

[1] Britt Turkot, Steven L. Carson, Anna Lio, Ted Liang, Mark Phillips, Brian McCool, Eric Stenehjem, Tim Crimmins, Guojing Zhang, Sam Sivakumar, "EUV progress toward HVM readiness", Proc. SPIE 9776, Extreme Ultraviolet (EUV) Lithography VII, 977602 (March 18, 2016); doi: 10.1117/12.2225014

[2] Noreen Harned, Roel Moors, Maarten van Kampen, Vadim Banine, Jeroen Huijbregtse, Roel Vanneer, Antoine Kempen, Dirk Ehm, Rogier Verberk, Edwin te Sligte, Arnold Storm, "Strategy for Minimizing EUV Optics", EUVL Symposium, (September 29 - October 2, 2008), Lake Tahoe

[3] Edwin te Sligte, Norbert Koster, Alex Deutz, Wilbert Staring, "A New Mask Exposure and Analysis Facility", in Proc. SPIE 9235, Photomask Technology 2014, 92351F (8 October 2014); doi: 10.1117/12.2083713

[4] Edwin te Sligte, Norbert Koster, Freek Molkenboer, Alex Deutz, "EBL2, a flexible, controlled EUV exposure and surface analysis facility", Photomask Japan (2016), DOI: 10.1117/12.2240302

[5] Edwin te Sligte, Norbert Koster, Freek Molkenboer, Peter van der Walle, Pim Muilwijk, Wouter Mulckhuyse, Bastiaan Oostdijck, Christiaan Hollemans, Björn Nijland, Peter Kerkhof, Michel van Putten, André Hoogstrate, Alex Deutz, "EBL2: high power EUV exposure facility”, Photomask Technology (2016), Proc. of SPIE Vol. 9985, 998520-1, doi: 10.1117/12.2240921

[6] Yusuke Teramoto, Bárbara Santos, Guido Mertens, Ralf Kops, Margarete Kops, Alexander von Wezyk, Hironobu Yabuta, Akihisa Nagano, Takahiro Shirai, Noritaka Ashizawa, Kiyotada Nakamura, Kunihiko Kasama, "Highradiance LDP source for mask-inspection application," in Extreme Ultraviolet (EUV), Lithography VI, Proc. of SPIE Vol. 9422 (SPIE, San Jose, CA, March 2015), pp. 94220F-1-9 